\section{ORIGINAL RESEARCH}

C. Brekenfeld

G. Schroth

P. Mordasini

U. Fischer

M.-L. Mono

A. Weck

M. Arnold

M. El-Koussy

J. Gralla

\title{
Impact of Retrievable Stents on Acute Ischemic Stroke Treatment
}

BACKGROUND AND PURPOSE: Retrievable stents combine the high recanalization rate of stents and the capability of removing the thrombus offered by mechanical thrombectomy devices. We hypothesized that retrievable stents shorten time to recanalization in the multimodal approach for endovascular stroke treatment.

MATERIALS AND METHODS: Forty consecutive patients with acute ischemic stroke and undergoing endovascular therapy were included. Treatment included thromboaspiration, thrombus disruption, thrombolysis, PTA, and stent placement. In 17 patients, a retrievable stent was used (group A) in addition to multimodal therapy. The remaining 23 patients constituted group B. Baseline characteristics, occlusion sites, urokinase dose, recanalization rate, and time to recanalization were compared between the groups.

RESULTS: Median NIHSS scores were higher in group A compared with group B on admission (19 versus $12.5 ; P=.018$ ) but were not significantly different at day 1 (14 versus $10 ; P=.6$ ). Intra-arterial thrombolysis was used in significantly fewer patients of group A than group B (53\% versus $87 \%$, respectively; $P=.017)$, and median urokinase dose was lower in group $A$ than in group $B(250,000 \mathrm{IU}$ versus $700,000 \mathrm{IU} ; P=.006$ ). Time to recanalization was significantly shorter in group A compared with group $B$ (median time to recanalization 52.5 minutes versus 90 minutes, respectively; $P=.001$ ). Recanalization rate was higher in group A than group B $(94 \%$ versus $78 \% ; P=.17)$.

CONCLUSIONS: Addition of retrievable stents to the multimodal endovascular approach for acute ischemic stroke treatment significantly reduces time to recanalization and further increases the recanalization rate.

ABBREVIATIONS: IQR = interquartile range; IU = internationals unit(s); $\mathrm{mRS}=$ modified Rankin Scale; NIHSS = National Institutes of Health Stroke Scale; PTA = percutaneous transluminal balloon angioplasty; TIMI = Thrombolysis in Myocardial Infarction

E arly recanalization reduces mortality and improves outcome in acute ischemic stroke patients. ${ }^{1}$ Endovascular revascularization approaches yield higher recanalization rates than intravenous thrombolysis, which is still the first-line evidence-based treatment for most acute ischemic stroke patients. In recent years, interest in mechanical thrombectomy has markedly increased, and numerous devices have been advocated for endovascular stroke treatment. ${ }^{2-4}$ Placement of an intracranial stent that compresses the occluding thromboembolus at the arterial wall is feasible and results in fast and efficient recanalization. ${ }^{5-7}$ However, permanent stent placement necessitates the additional application of antiplatelet agents, ie, aspirin and clopidogrel. To avoid the risk of hemorrhage after antiplatelet therapy and the risk of in-stent stenosis, some groups have just placed stents temporarily. ${ }^{8-13}$ The concept of a retrievable stent combines the fast and efficient recanalization effect of an intracranial stent and the capability of extracting the thromboembolus offered by mechanical thrombectomy devices.

Received September 3, 2010; accepted after revision November 23

From the University Institute of Diagnostic and Interventional Neuroradiology (C.B., G.S., P.M., M.E.-K., J.G.), Inselspital, Bern, Switzerland; and Department of Neurology (U.F., M.-L.M., A.W., M.A.), Inselspital, Bern, Switzerland

Please address correspondence to Caspar Brekenfeld, MD, University Institute of Diagnostic and Interventional Neuroradiology, Inselspital, University of Bern, Freiburgstr 4, CH-3010 Bern, Switzerland; e-mail, caspar.brekenfeld@insel.ch

Indicates open access to non-subscribers at www.ajnr.org

DOI 10.3174/ajnr.A2494
In our department, endovascular stroke treatment has been performed since 1992 and includes thromboaspiration, intra-arterial thrombolysis, and mechanical thrombectomy as well as PTA and stent placement. ${ }^{6,14-16}$ We have used retrievable stents in addition to the established techniques since January 2010. This study evaluates the impact of the use of a retrievable stent on multimodal endovascular stroke treatment. We hypothesized that the application of a retrievable stent accelerates time to recanalization during endovascular stroke treatment.

\section{Materials and Methods}

The endovascular treatment of acute ischemic stroke was approved by our local ethics committee. Data of all stroke patients are registered prospectively in our stroke data base. For this study, all acute ischemic stroke patients who were treated with endovascular therapy during a 5-month period (November 2009-April 2010) were analyzed. The inclusion criteria for endovascular treatment were as follows: 1) clinical diagnosis of acute stroke established by a stroke neurologist; 2) acute neurologic deficit and corresponding cerebral artery occlusion; 3) baseline NIHSS score $\geq 4$, except for isolated aphasia or hemianopsia; 4) exclusion of cerebral hemorrhage by CT or MR imaging; 5) for patients $>75$ years old, their general condition before stroke did not advise against it; and 6) informed consent of patient or next of kin.

The treatment consisted of 1) thromboaspiration, 2) intra-arterial thrombolysis, 3) mechanical thrombus disruption, 4) mechanical thrombectomy, and 5) PTA and/or stent implantation. Intra-arterial urokinase (Urokinase Medac; Medac, Wedel, Germany) was administered up to a dose of $1000000 \mathrm{IU}$ in the first 6 hours, whereas 
thromboaspiration and mechanical thrombectomy were performed up to 8 hours after symptom onset. Treatment also was performed beyond 8 hours in selected patients who presented with small areas of infarct on diffusion-weighted imaging, large hypoperfused areas on perfusion-weighted imaging, and persistent occlusion of a large cerebral artery.

All patients were evaluated on admission by an experienced neurologist and assessed according to the NIHSS. Patients treated with combined intravenous thrombolysis/endovascular approach also were included.

\section{Endovascular Treatment}

Endovascular treatments were performed by 1 or 2 neuroradiologists (C.B., J.G., G.S.). After placement of an $8 \mathrm{~F}$ catheter sheath in the femoral artery, a 4-vessel diagnostic angiography was performed to assess vessel occlusion and collateral flow to the infarcted area. After confirmation of vessel occlusion, an $8 \mathrm{~F}$ guide catheter (Guider Softip; Boston Scientific, Natick, Massachusetts) or a balloon guide catheter (Merci 8F Balloon Guide Catheter; Concentric Medical, Mountain View, California) was introduced into the target cervical artery.

For thromboaspiration, the guide catheter was introduced as far as possible (eg, into the petrous segment of the internal carotid artery), and the occlusion site was approached by using a $5.1 \mathrm{~F}$ aspiration catheter (Vasco35+ASPI; Balt, Montmorency, France). If necessary, an additional microcatheter (2.4F Renegade or 2.8F Renegade Hi-Flo, Boston Scientific or 2.3F Prowler Select Plus, Cordis, Miami Lakes, Florida) was introduced into the Vasco35+ASPI (triaxial catheterization technique) to overcome tortuous vessel segments such as the carotid siphon. This technique even allows for advancement of the Vasco35+ASPI into the distal mainstem of the middle cerebral artery. Aspiration was created by $60-\mathrm{mL}$ syringes connected to the Vasco35+ASPI as well as to the guide catheter. The Vasco35+ASPI was removed during continuous aspiration at both sites.

Mechanical thrombus disruption was performed either during intra-arterial thrombolysis, consisting of gentle maneuvers with the microcatheter and/or the microwire (SilverSpeed 14; ev3 Endovascular, Plymouth, Minnesota) at the thrombus, which was repeatedly passed if possible, or by using the Penumbra system (Penumbra, Alameda, California). This latter method included continuous aspiration in front of the thrombus (Reperfusion Catheter 041; Penumbra) while the thrombus was disrupted with the separator wire (Separator 041; Penumbra).

Intra-arterial thrombolysis was applied via a microcatheter for 60-90 minutes. The microcatheter tip was placed in the occluding thrombus. Follow-up angiograms were acquired every 20-30 minutes to assess recanalization and to terminate thrombolysis in the case of recanalization.

For intracranial stent placement, the thrombus/occlusion was passed with a microcatheter and a stent delivery catheter (Wingspan stent system; Boston Scientific) was advanced over a long exchange wire (Transcend 300; Boston Scientific). Extracranial carotid stents (Cristallo Ideale Carotid Stent System, Invatec, Brescia, Italy; or Precise, Cordis) were placed under fluoroscopic control while retracting the $8 \mathrm{~F}$ guide catheter into the common carotid artery. Distal protection (FilterWire EZ; Boston Scientific) was used during carotid stent placement. An Avion Plus PTA balloon catheter (Invatec, Roncadelle, Italy) was used for PTA. Generally, $500 \mathrm{mg}$ of aspirin was given intravenously if a stent had to be deployed permanently. Balloon angioplasty was performed in the case of residual stenosis or in-stent thrombus formation.
The Solitaire FR Revascularization Device (ev3) was used for mechanical thrombectomy. This device consists of a self-expandable nitinol stent firmly mounted at the push wire. Because the stent is not detached, it can be retrieved even after complete deployment. All retrievable stents used in this study measured $4 \mathrm{~mm}$ in diameter and $20 \mathrm{~mm}$ in length. First, the thrombus was passed with the microcatheter (2.3F Prowler Select Plus), and a distal angiogram was performed to confirm proper localization in the target cerebral artery. The Solitaire FR Revascularization Device was used by retraction of the microcatheter and placed at the occlusion site under fluoroscopic control, immediately followed by an angiogram to assess recanalization. Considering the results of a previously performed animal study by using the Solitaire FR Revascularization Device in 15 vessel occlusions, ${ }^{17}$ we left the stent in place for 5 minutes before the microcatheter was advanced to resheath the connection site of the stent at the push wire. This maneuver stabilizes the connection zone and reduces the risk of device fracture. Both the stent and the microcatheter were retrieved simultaneously during aspiration at the guide/balloon catheter. Flow arrest was applied at the discretion of the interventionalist. In the case of failed or insufficient recanalization, the Solitaire FR Revascularization Device was cleaned and reinserted. Up to 3 retrieval attempts were performed per occlusion.

Follow-up angiography was performed after each revascularization attempt with the above-mentioned endovascular techniques to assess the recanalization results as well as thromboembolic events to a previously unaffected vessel territory, eg, occlusion of anterior cerebral artery after thrombus retrieval from middle cerebral artery. The recanalization rate was assessed according to the TIMI grades. ${ }^{18}$ TIMI grades 2 and 3 were rated as sufficient recanalization, whereas TIMI grades 0 and 1 were rated as insufficient recanalization.

The endovascular procedures were performed during general anesthesia or with an anesthesia stand-by team. Patients were observed for at least 24 hours in an intensive care unit, and CT was performed in cases of clinical worsening to rule out intracranial hemorrhage. The NIHSS score at day 1 was assessed by our stroke neurologists. Clinical outcome was assessed by a neurologist at 3 months according to the mRS. ${ }^{19}$ For statistical evaluation, mRS scores were dichotomized to good outcome (mRS 0-2) and poor outcome (mRS 3-6).

Different time points were recorded for this analysis: symptom onset, MR imaging or CT study before intervention, first angiogram, achievement of main recanalization, and final angiogram. Time intervals from the first angiogram to the main recanalization (time to recanalization) were calculated. Main recanalization was defined as sufficient recanalization (TIMI 2/3) and flow to the ischemic territory.

For comparison, patients were stratified into 2 groups: group A comprised all patients treated with the Solitaire FR Revascularization Device, and group B comprised all patients who were not. Group comparisons were performed by using the $t$ test for continuous and the Mann-Whitney $\mathrm{U}$ test for noncontinuous variables, and $P$ values $<.05$ were considered statistically significant.

\section{Results}

During the 5-month period (November 2009-April 2010), 40 consecutive patients (15 women; median age, 67 years [IQR, 59-77]) were treated by endovascular means for acute ischemic stroke. Occlusions were located in the internal carotid artery $(n=12)$, the M1 segment of middle cerebral artery $(n=$ $17)$, the M2 segment $(n=7)$, and the basilar artery $(n=4)$. 


\begin{tabular}{|c|c|c|c|}
\hline & Group A & Group B & $P$ Value \\
\hline Age (yr) (median [IOR]) & $66(61-77)$ & $68(57.5-78)$ & NS \\
\hline Sex (\%), female & 41 & 35 & NS \\
\hline NIHSS score, median (IOR) & $19(14-22)$ & $12.5(10-15.5)$ & .018 \\
\hline Arterial hypertension (\%) & 65 & 65 & NS \\
\hline Diabetes mellitus (\%) & 18 & 17 & NS \\
\hline Hypercholesterolemia (\%) & 71 & 65 & NS \\
\hline Nicotine abuse (\%) & 18 & 17 & NS \\
\hline Intravenous thrombolysis (\%) & 35 & 22 & NS \\
\hline Multimodal therapy (\%) & 76 & 74 & NS \\
\hline Thrombaspiration (\%) & 41 & 61 & NS \\
\hline Intra-arterial thrombolysis & 53 & 87 & .017 \\
\hline Urokinase dose (IU), median (IQR) & $250000(0-500000)$ & 700000 (475 000-1 000000$)$ & .006 \\
\hline Stent/PTA $(\%)$ & 24 & 35 & NS \\
\hline Intubation (\%) & 88 & 70 & NS \\
\hline Recanalization TIMI 2/3 (\%) & 94 & 78 & NS \\
\hline Time to recanalization, min (median [IOR]) & $52.5(37.5-61)$ & $90(66-112.5)$ & 0.001 \\
\hline NIHSS score $1 \mathrm{~d}$, median (IOR) & $14(6-20)$ & $10(6-20)$ & NS \\
\hline Good outcome (\%; mRS 0-2) at 3 mo follow-up & 43 & 52 & NS \\
\hline
\end{tabular}

The median NIHSS score on admission was 15.5 (IQR, 12-20).

Patient characteristics and demographic factors of both groups are presented in the Table. Stroke severity on admission differed significantly between groups (group A: median NIHSS score 19; IQR, 14-22; group B: median NIHSS score 13; IQR, $10-15.5 ; P=.018$ ). The groups did not differ with respect to occlusion site (internal carotid artery, $n=5$ versus $n=7$ for group A and group B, respectively; M1 segment, $n=$ 7 versus $n=10$; M2 segment, $n=2$ versus $n=5$; basilar artery, $n=3$ versus $n=1$ ) or the treatment modalities applied (other than the use of the Solitaire FR Revascularization Device; Table). Time to recanalization was significantly shorter in group A (median, 52.5 minutes; IQR, 37.5-61.0 minutes) compared with group B (median, 90.0 minutes; IQR, 66.0-112.5 minutes; $P=.001$ ). Sufficient recanalization (TIMI 2/3) was achieved in group A in $94.1 \%$ of patients compared with $78.3 \%$ in group $\mathrm{B}(P=.179)$.

Delivery of the Solitaire FR Revascularization Device was technically feasible in all patients of group A. The retrievable stent was used as the exclusive endovascular treatment in 4 patients. Flow arrest was applied during stent retrieval in 12 patients. Only 1 retrieval attempt with the stent was performed in 13 patients. Two retrieval attempts were performed in 2 patients and 3 retrieval attempts in another 2 patients. Overall, 23 retrieval attempts were performed. Immediate recanalization after deployment of the Solitaire FR Revascularization Device was observed in 18/23 (78\%) attempts (Fig 1). Retrieval of the stent resulted in sufficient recanalization in 14/17 patients (82\%). In 12 patients (71\%), thrombotic material was removed and normally found attached to the stent meshes (Fig 1). No thromboembolic occlusion of a previously unaffected artery was found at follow-up angiography after retrieval. In 2 of 3 patients with failed recanalization after removal of the Solitaire FR Revascularization Device, the vessel was finally recanalized by permanent placement of an intracranial stent (Wingspan). Overall, 35\% of the patients in group A received intravenous thrombolysis, 53\% intra-arterial thrombolysis, $41 \%$ thromboaspiration, and $24 \%$ an additional stent place- ment with or without PTA (2 stent placements into intracranial arteries and 2 stent placements into the proximal internal carotid artery). Eighty-eight percent of the interventions in group A were performed during general anesthesia. No symptomatic intracranial hemorrhages or device-related adverse events were encountered.

In group $\mathrm{B}, 22 \%$ of the patients received intravenous thrombolysis, $87 \%$ intra-arterial thrombolysis, $61 \%$ thromboaspiration, and $35 \%$ stent placement with or without PTA (3 stent placements into intracranial arteries and 5 stent placements into the proximal internal carotid artery). Intra-arterial thrombolysis was used as the exclusive endovascular treatment in 6 patients of this group (26\%). General anesthesia during endovascular treatment was applied in $70 \%$ of patients. Intra-arterial urokinase was used significantly more often in group B than group A ( $87 \%$ versus 53\%, respectively; $P=$ $.017)$, and the median urokinase dose was significantly higher $(P=.006$; Table) in group B (median dose, 700000 IU; IQR, $475000-1000000$ ) than in group A (median dose, 250000 IU; IQR, 0-500 000). Clinical outcome assessed according to the NIHSS score at day 1 and to the mRS at 3 months did not differ between groups.

\section{Discussion}

Early recanalization is associated with better outcomes and lower levels of mortality in acute ischemic stroke patients. ${ }^{1}$ Recanalization rates of endovascular treatment are already considerably high, with a range of $66 \%-76 \%$ for intra-arterial thrombolysis ${ }^{15,20}$ and $68 \%-81.6 \%$ for the combination of mechanical thrombectomy with intravenous and intra-arterial thrombolysis. ${ }^{2,3}$ However, intra-arterial thrombolysis is sometimes a long procedure, normally lasting $60-120 \mathrm{~min}$ utes, and the mean time to recanalization in one study by using the Penumbra system was $97 \pm 37$ minutes. ${ }^{21}$ New devices for acute stroke treatment, such as the Solitaire FR Revascularization Device, should have recanalization rates at least as high as the above-mentioned combined therapies. Ideally, and perhaps more importantly, these devices should reduce time to recanalization. The present study verified that the use of a 


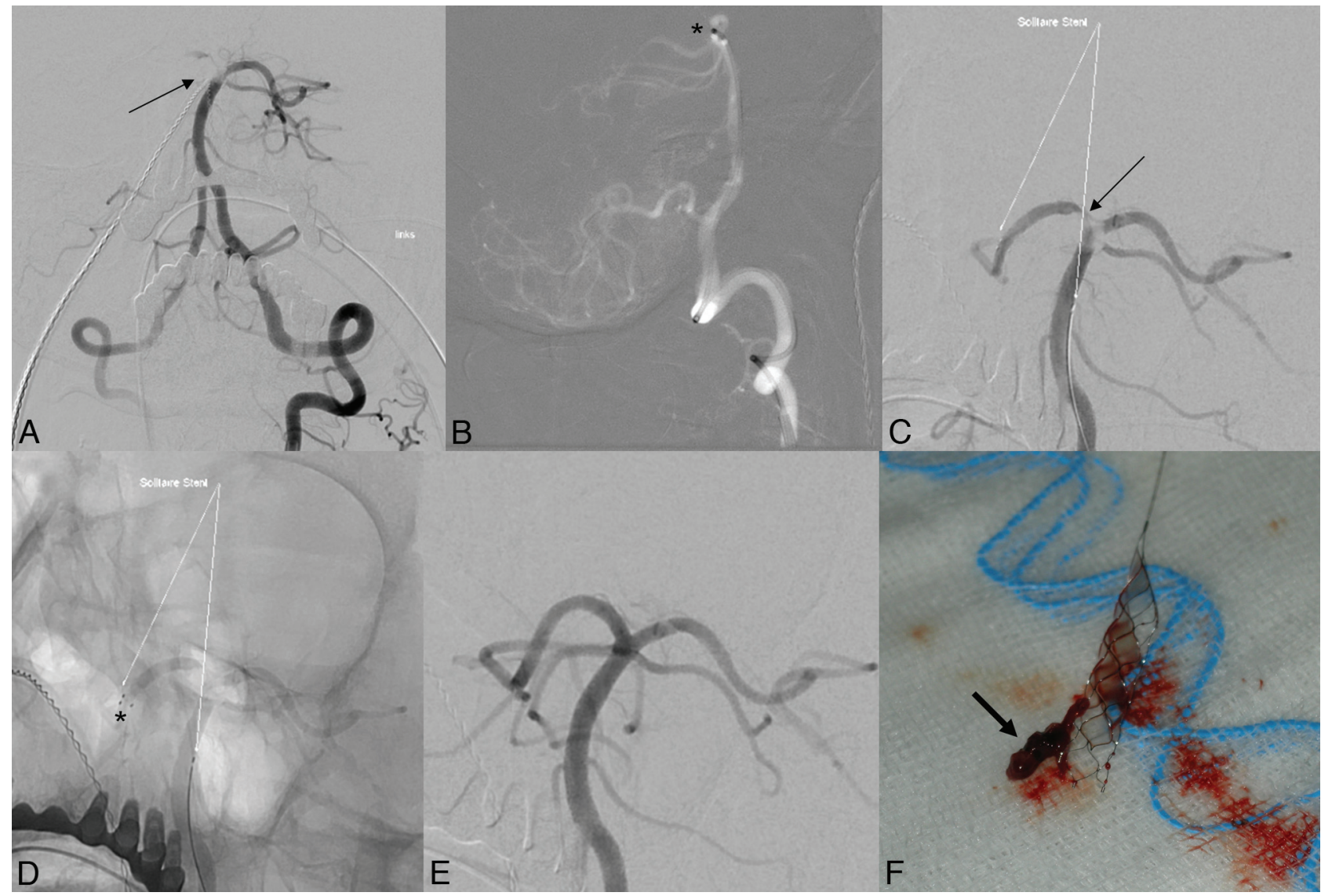

Fig 1. Occlusion of the tip of the basilar artery. Symptom onset $>12$ hours before angiography. NIHSS score 6 . A, Antero-posterior view of left vertebrobasilar angiogram depicts thrombus in the distal basilar artery, residual flow to left posterior cerebral artery, occlusion of right posterior cerebral artery and right superior cerebellar artery. $B$, Thromboaspiration attempt-tip of Vasco35+ASPI (*) in the tip of basilar artery (lateral view). C, After unsuccessful thromboaspiration, deployment of a Solitaire FR 4/20. D, Distal stent markers in the right posterior cerebral artery. The proximal stent ends in the basilar artery. Note incomplete recanalization of the right posterior cerebral artery after stent placement. $E$, Complete recanalization after stent retrieval. F, Solitaire FR Device with captured thrombus (arrow). Time to recanalization 20 minutes.

retrievable stent significantly reduces the time to recanalization in a multimodal approach to endovascular stroke treatment. Because ischemic penumbral tissue irreversibly turns into damaged brain tissue over time, ${ }^{22,23}$ faster recanalization should result in less infarct growth and better outcome. We observed a more pronounced reduction of the NIHSS score during the first 24 hours after stroke onset in patients of group A compared with group $\mathrm{B}$, which might be explained by the significantly faster recanalization achieved in group A. However, further studies are needed to assess the long-term effect of this improved endovascular treatment on patient outcome. In addition, the recanalization rate of the multimodal approach including the Solitaire FR Revascularization Device was higher compared with the multimodal approach without the use of the retrievable stent. Due to the small number of patients included, this result did not reach significance.

The implantation of an intracranial stent is very effective for recanalization in acute ischemic stroke. ${ }^{5-7}$ However, permanent stent implantation carries the risk of in-stent thrombosis, and it requires dual antiplatelet therapy, which increases the risk of hemorrhage. In addition, restenosis and reocclusion rates between $25 \%$ and $32.3 \%$ have been reported. ${ }^{24,25}$ To avoid these problems, temporary stent placement with stent retrieval at the end of the intervention seems to be a promising approach. Cerebral vessel recanalization with the temporary placement of an intracranial stent in conjunction with throm- bolysis and/or mechanical thrombectomy was first reported in 4 cases, including the use of the Enterprise stent (Cordis) $(n=$ $3)$ and the Solitaire AB Device $(n=1) \cdot{ }^{8-11}$ Both devices had originally been introduced as detachable remodeling stents for aneurysm treatment. Only the nondetachable Solitaire FR Revascularization Device was used in the present study.

The Solitaire FR Revascularization Device was successfully integrated into our multimodal endovascular approach for acute ischemic stroke treatment. It was applied with a high technical success rate, and we observed no device-related adverse events confirming the findings of 2 previously published studies. ${ }^{12,13}$ However, during this study the sequence of the different endovascular approaches differed: thromboaspiration was the first-line treatment in most patients followed by intra-arterial thrombolysis or mechanical thrombectomy with the Solitaire FR Revascularization Device, whereas mechanical thrombectomy with the Solitaire FR Revascularization Device was the first-line treatment followed by intra-arterial thrombolysis in some patients with residual peripheral occlusion. From the results of this study, it can be assumed that the use of a retrievable stent as the first-line treatment in all patients might result in an even shorter time to recanalization than the reported 52.5 minutes. Castano et $\mathrm{al}^{12}$ reported a median time from groin puncture to recanalization of 50 minutes when the retrievable stent was the first endovascular approach. The recanalization rate achieved with the Solitaire FR Revasculariza- 
tion Device was $82 \%$. Remarkably, thrombi were retrieved in $71 \%$ of the patients, demonstrating the capability of the retrievable stent as a thrombectomy device. If recanalization fails, further techniques can be applied, for example, intraarterial thrombolysis or permanent stent placement. The combination of a retrievable stent with other endovascular techniques yielded a recanalization rate of $94 \%$ comparable with recently published results of $90 \%$ and $90.9 \% .^{12,13}$

Remarkably, significantly fewer patients were treated with intra-arterial urokinase and lower doses of urokinase were given in the group of patients treated with the retrievable stent compared with the group of patients treated without the retrievable stent. Higher urokinase doses are a predictor of symptomatic intracerebral hemorrhage. ${ }^{26}$ Therefore, a less frequent use and lower dose of urokinase should result in lower rates of symptomatic intracerebral hemorrhage in patients who are treated with the retrievable stent. However, due to the small sample sizes the risk of symptomatic intracerebral hemorrhage could not be evaluated in this study.

Thromboembolic complications resulting in occlusion of previously unaffected arteries after mechanical thrombectomy are a major concern. However, no such complications were encountered in this study confirming previously reported data. ${ }^{12,13}$ This finding might be explained by the use of flow arrest and aspiration during retrieval of the Solitaire FR Revascularization Device. A balloon guide catheter was used in most patients and in 20 patients treated by Castano et al. ${ }^{12}$ However, in 22 patients treated without balloon occlusion no thromboembolic events were encountered as well. ${ }^{13}$

This study did not assess the long-term outcomes of the patients. However, a trend for improved outcome in the patients who were treated with the retrievable stent might be reflected by the short-term evolution of the NIHSS scores in both groups. Group A patients (multimodal stroke treatment including the retrievable stent) had more severe strokes compared with group B (multimodal treatment without retrievable stent), but at day 1 the NIHSS scores were no longer significantly different between the 2 groups.

\section{Conclusions}

The use of a retrievable stent improved the recanalization rate of the multimodal endovascular revascularization approach and, more importantly, significantly reduced the time needed for recanalization. Faster recanalization is expected to result in less infarct growth. No technical failures or device-related adverse events were encountered.

\section{Acknowledgments}

We thank Barbara Catherine Ritter, MSc, for statistical advice.

\section{References}

1. Rha JH, Saver JL. The impact of recanalization on ischemic stroke outcome: a meta-analysis. Stroke 2007;38:967-73
2. Smith WS, Sung G, Saver J, et al. Mechanical thrombectomy for acute ischemic stroke: final results of the Multi MERCI trial. Stroke 2008;39:1205-12

3. The Penumbra Pivotal Stroke Trial Investigators: the Penumbra pivotal stroke trial: safety and effectiveness of a new generation of mechanical devices for clot removal in intracranial large vessel occlusive disease. Stroke 2009;40:2761-68

4. Brekenfeld C, Schroth G, El-Koussy M, et al. Mechanical thromboembolectomy for acute ischemic stroke: comparison of the Catch thrombectomy device and the Merci retriever in vivo. Stroke 2008;39:1213-19

5. Levy EI, Mehta R, Gupta R, et al. Self-expanding stents for recanalization of acute cerebrovascular occlusions. AJNR Am J Neuroradiol 2007;28:816-22

6. Brekenfeld C, Schroth G, Mattle HP, et al. Stent placement in acute cerebral artery occlusion: use of a self-expandable intracranial stent for acute stroke treatment. Stroke 2009;40:847-52

7. Zaidat OO, Wolfe T, Hussain SI, et al. Interventional acute ischemic stroke therapy with intracranial self-expanding stent. Stroke 2008;39:2392-95

8. Kelly ME, Furlan AJ, Fiorella D. Recanalization of an acute middle cerebral artery occlusion using a self-expanding, reconstrainable, intracranial microstent as a temporary endovascular bypass. Stroke 2008;39:1770-73

9. Suh SH, Lee KY, Hong CK, et al. Temporary stenting and retrieval of the self-expandable, intracranial stent in acute middle cerebral artery occlusion. Neuroradiology 2009;51:541-44

10. Castano C, Serena J, Davalos A. Use of the new Solitaire ${ }^{\mathrm{TM}} \mathrm{AB}$ device for mechanical thrombectomy when Merci clot retriever has failed to remove the clot. Interventional Neuroradiology 2009;15:109-214

11. Hauck EF, Mocco J, Snyder KV, et al. Temporary endovascular bypass: a novel treatment for acute stroke. AJNR Am J Neuroradiol 2009;30:1532-33

12. Castano C, Dorado L, Guerrero C, et al. Mechanical thrombectomy with the Solitaire $\mathrm{AB}$ device in large artery occlusions of the anterior circulation: a pilot study. Stroke 2010;41:1836-40

13. Roth C, Papanagiotou $P, B e h n k e ~ S$, et al. Stent-assisted mechanical recanalization for treatment of acute intracerebral artery occlusions. Stroke 2010;41:2559-67

14. Gonner F, Remonda L, Mattle H, et al. Local intra-arterial thrombolysis in acute ischemic stroke. Stroke 1998;29:1894-900

15. Arnold M, Schroth G, Nedeltchev K, et al. Intra-arterial thrombolysis in $\mathbf{1 0 0}$ patients with acute stroke due to middle cerebral artery occlusion. Stroke 2002;33:1828-33

16. Nedeltchev K, Brekenfeld C, Remonda L, et al. Stenting of the internal carotid artery in acute stroke: preliminary results of 25 patients. Radiology 2005;237:1029-37

17. Mordasini P, Frabetti N, Schroth G, et al. In vivo evaluation of the first dedicated combined flow-restoration and mechanical thrombectomy device in a swine model of acute vessel occlusion. AJNR Am J Neuroradiol 2011;32:294-300

18. The Thrombolysis in Myocardial Infarction (TIMI) trial. Phase I findings. TIMI Study Group. N Engl J Med 1985;312:932-36

19. Van Swieten JC, Koudstaal PJ, Visser MC, et al.. Interobserver agreement for the assessment of handicap in stroke patients. Stroke 1988;19:604-07

20. Furlan A, Higashida R, Wechsler L, et al.. Intra-arterial proUK for acute ischemic stroke. The PROACT II study: a randomized controlled trial. Prolyse in acute cerebral thromboembolism. JAMA 1999;282:2003-11

21. Kulcsár Z, Bonvin C, Pereira VM, et al. Penumbra system: a novel mechanical thrombectomy device for large-vessel occlusions in acute stroke. AJNR Am J Neuroradiol 2010;31:628-33

22. Darby DG, Barber PA, Gerraty RP, et al. Pathophysiological topography of acute ischemia by combined diffusion-weighted and perfusion MRI. Stroke 1999;30:2043-52

23. Markus R, Reutens DC, Kazui S, et al. Topography and temporal evolution of hypoxic viable tissue identified by $18 \mathrm{~F}$-fluoromisonidazole positron emission tomography in humans after ischemic stroke. Stroke 2003;34:2646-52

24. Albuquerque FC, Levy EI, Turk AS, et al. Angiographic patterns of Wingspan in-stent restenosis. Neurosurgery 2008;63:23-27

25. Zaidat OO, Klucznik R, Alexander MJ, et al. NIH Multi-center Wingspan Intracranial Stent Registry Study Group. The NIH registry on use of the Wingspan stent for symptomatic 70-99\% intracranial arterial stenosis. Neurology 2008;70:1518-24

26. Brekenfeld C, Remonda L, Nedeltchev K, et al. Symptomatic intracranial haemorrhage after intra-arterial thrombolysis in acute ischaemic stroke: assessment of 294 patients treated with urokinase. J Neurol Neurosurg Psychiatry 2007;78:280-85 